\title{
Downstream GPCR inhibition
}

DOI:

10.1038/nrd2181
Although G-protein-coupled receptors (GPCRs) and their downstream signalling pathways are involved in cancer progression, the therapeutic potential of antagonists acting at specific GPCRs is limited as tumour growth is driven by the simultaneous activation of several GPCRs. Now, Prévost and colleagues, writing in Cancer Research, have demonstrated an alternative approach to blocking GPCR-mediated signalling by developing a novel anticancer agent that selectively inhibits the heterotrimeric G-protein subunit complex.

By screening a chemical library, Prévost and colleagues identified a lead compound - BIM-46174 — that inhibited $\mathrm{G}_{\alpha}$-mediated adenylyl cyclase activation and $\mathrm{G}_{\mathrm{q}}{ }^{-}$ mediated increases in intracellular calcium. Next, the authors tested the capacity of BIM-46174 to inhibit cancer cell invasion induced by the Wnt oncogenic pathway, which is directly connected with $\mathrm{G}_{\alpha \mathrm{\alpha o} / \mathrm{i}}$ and $\mathrm{G}_{\beta \gamma}$ proteins. Treatment of Wnt2transformed human colon cancer cells with BIM-46174 significantly inhibited the invasive potential of these cells in collagen type- 1 gels, but had no effect on cancer cell invasion induced by individual subunits of the G-protein complex.

BIM-46174 was shown to have antiproliferative effects on human cancer cell lines established from numerous tumour sites. Although the potency of BIM-46174 was dependent on the exposure time, the

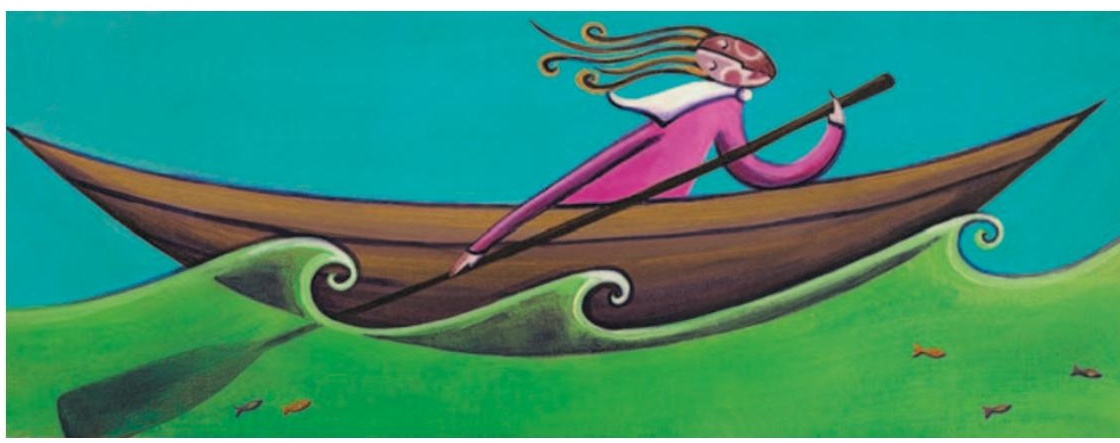

compound was active against several drug-resistant cell lines, independent of the resistance mechanism involved. The authors next assessed whether the antiproliferative effect of BIM-46174 was associated with alterations in lung cancer cell survival. Treatment with BIM-46174 led to a fivefold increase in apoptosis in two lung cancer cell lines.

In the National Cancer Institute COMPARE assay - a cell-growth inhibition assay designed to identify compounds with novel mechanisms of action - no correlation was found between BIM-46174 and 12,000 known active compounds, suggesting that the compound has a novel mechanism of action.

Finally, the anticancer effects of BIM-46174 were assessed in a mouse xenograft model using xenografts established from human small-cell lung cancer (SCLC), non-small-cell lung cancer (NSCLC) and pancreatic cancer cells. Combined treatment with BIM-46174 and the approved lung cancer agent cisplatin induced a drastic reduction of the tumour growth rate in SCLC and NSCLC, greater then that produced by either agent alone. Combining BIM-46174 with a farnesyltransferase inhibitor against SCLC, or with a topoisomerase inhibitor against pancreatic cancer, showed significant antitumour activity. Despite limited weight loss, there was no evidence of toxicity in a preliminary assessment.

Although BIM-46174 and related compounds have not yet been tested in clinical trials, this study provides evidence that an inhibitor of the heterotrimeric G-protein complex could be used to treat certain aggressive, hard-to-treat types of human cancer, such as lung and pancreatic tumours.

Charlotte Harrison

ORIGINAL RESEARCH PAPER Prévost, G. P. et al. Anticancer activity of BIM-46174, a new inhibitor of the heterotrimeric $\mathrm{G}_{\alpha} / \mathrm{G}_{\beta \gamma}$ complex. Cancer Res. 66, 9227-9234 (2006) 